\title{
The transition of pediatric Marfan patients to adult care: a challenge and its risks
}

\author{
Veronika C. Stark ${ }^{1}$, Katrin Doering ${ }^{1}$, Yskert von Kodolitsch ${ }^{2}$, Rainer Kozlik-Feldmann ${ }^{1}$, Götz C. \\ Mueller $^{1}$, Jakob Olfe ${ }^{1}$, Meike Rybczynski ${ }^{2}$, Helke Schueler ${ }^{2}$, Thomas S. Mir ${ }^{1}$ \\ ${ }^{1}$ Pediatric Cardiology, ${ }^{2}$ Cardiology, University Heart Center, Hamburg, Germany \\ Contributions: (I) Conception and design: VC Stark, TS Mir; (II) Administrative support: TS Mir, R Kozlik-Feldmann; (III) Provision of study \\ material or patients: None; (IV) Collection and assembly of data: K Doering, J Olfe, GC Mueller, TS Mir, R Kozlik-Feldmann, H Schueler, \\ M Rybczynski, Y von Kodolitsch; (V) Data analysis and interpretation VC Stark, K Doering; (VI) Manuscript writing: All authors; (VII) Final \\ approval of manuscript: All authors. \\ Correspondence to: Veronika C. Stark. Pediatric Cardiology, University Heart Center Hamburg-Eppendorf, Martinistrasse 52, 20246 Hamburg, \\ Germany. Email: ve.stark@uke.de.
}

Background: Care for patients with Marfan syndrome (MFS) has improved substantially in recent decades. Increasing clinical knowledge and genetic analysis allow early diagnosis of the disease in childhood. Because of the earlier initiation to preventive and medical treatment, patients' life expectancy has risen. To ensure optimal care, pediatric patients require a safe follow-up regime, multidisciplinary care, and a safe transition to adult care.

Methods: We collected a sample of 149 pediatric Marfan patients, of whom 34 patients had already been transferred to adult care or who were currently transitioning. First, we evaluated clinical aspects of patients that manifest in childhood and are present in the transition process. Second, we analyzed the transition process itself.

Results: We found age-dependent manifestation of organ pathologies. Dilatation of the sinus of Valsalva showed a particularly high prevalence during the transition process and $62 \%$ of patients required medical treatment. Mean onset of aortic root dilatation was $9.9 \pm 5.8$ years. Concerning systemic manifestation in MFS skin striae, wrist and thumb sign, and reduced elbow extension occurred significantly more often in patients who were transitioning than in younger children with MFS. All other clinical Marfan features showed an increased prevalence in patients who were transitioning compared with younger patients. In our cohort, transition was successful in 20 patients (58.9\%), 12 patients (35.3\%) are still in the transition process and 2 patients $(5.9 \%)$ were lost to follow up.

Conclusions: Marfan patients in the transition process are already under a chronic disease condition with a high onset of especially cardiovascular pathologies. Although early medical treatment in childhood is effective, the pathologies of the connective tissue require lifelong attention and influence life in many ways. The big challenge during transition is the double change of responsibility from the parents and pediatric doctor to the patient and adult doctor. Consequently, patients in transition process require special attention and close contact with the doctor and the family. A reevaluation by the supervising pediatric Marfan specialist of the successful transition to adult care is indispensable before the pediatric care of Marfan patients is completed.

Keywords: Aortic dilatation; Marfan syndrome (MFS); medical treatment; systemic manifestation; transition process

Submitted Jul 20, 2018. Accepted for publication Sep 19, 2018.

doi: $10.21037 / \mathrm{cdt} .2018 .09 .13$

View this article at: http://dx.doi.org/10.21037/cdt.2018.09.13 


\section{Introduction}

Marfan syndrome (MFS) is an autosomal-dominantly inherited disorder with a variable phenotype. It requires lifelong multidisciplinary medical involvement in all patients (1). It appears with a wide range from $1.5-17.2$ per 100,000 , or $0.075-0.86$ per 5,000 individuals in the general population and is a typical rare disease (2-4). Diagnosis of MFS is based on the 2010 revised Ghent Criteria (RGC), which includes clinical and genetic analyses (5). Because of the age-dependent onset of Marfan features and similarity of phenotype aspects to non-pathological pediatric standard variants, diagnosis and disease exclusion has always been even more challenging in children than in adults (6). However, advances in genetic analysis have allowed for earlier diagnosis of disease. In cases with a positive family history, genetic analysis enables diagnosis of MFS before clinical features appear. Approximately $75 \%$ of pediatric patients have an affected parent with MFS. Only $25 \%$ are diagnosed with a de novo mutation (7). Consequently, the number of patients who are diagnosed with MFS in childhood rises.

Appropriate medical interventions such as administration of pharmacological treatment (sartans and beta-blockers) and aortic surgery have extended the life expectancy of Marfan patients $(8,9)$. Today, preventive rather than reactive medicine is preferred and should start as early as possible. Therefore, physical medical treatment and psychological support for young patients is important. A continuous disease education in pediatric patient care is indispensable to enable patients to cope with the emotional distress of disease (10).

In summary, there is a growing cohort of pediatric Marfan patients and a need of early preventive medicine to extend their life expectancy. Patients are diagnosed early and require a lifelong, individualized follow-up and consequently a transition to adult care when they reach adulthood. Therefore, a safe transition process for consistently following-up examinations and multidisciplinary care are vital for optimal treatment.

We hereby present more than 10 years of experience with pediatric MFS and transition to adult care. First, we analyzed the clinical condition of pediatric Marfan patients undergoing transition. We concentrated on Marfan features that were relevant to the transition process and the need for pharmacological and surgical treatment. Second, we analyzed the transition process itself. We hereby describe general and individual problems in the changeover to adult care.

\section{Methods}

From January 2008 through October 2017, we investigated all patients with suspected MFS visiting our specialized pediatric Marfan Clinic at University Heart Center Hamburg. We examined all patients according to the RGC. Genetic analysis was initialized as soon as diagnosis of MFS was reasonable according to clinical examination or family history. Additionally, we performed MRI whenever possible and useful.

We defined transition as the change of care from the pediatric doctors to the adult doctors. Thereby also patients are increasingly taking responsibility for their disease instead of their parents whereas an objective presentation of this is difficult. Thus this analysis concentrates on the change of the professional medical care. The period of transition starts with a preparing process of pediatric patients and their families around the age of 16 years. Transition process to adult care itself was performed with an age between 18 and 21 years. A successful transition was defined as the first presentation to the specialized adult Marfan Clinic of the cardiology department in University Heart Center Hamburg. In our cohort the transition process was completed around an age of 19 to 24 years.

We analyzed all Marfan features in our group of patients before and after transition process, which implies patients younger than 18 and patients older than 18 years. Concerning the young adults group only patients in transition progress were included. In consequence the oldest patient was 24 years old. We evaluated the pharmacological and surgical treatment in all patients. Concerning pharmacological treatment in childhood, in first line we started sartan treatment. We administered Valsartan two times a day at a target dose of $2 \mathrm{mg}$ per kilogram of body weight per day. Therapy was started with a dose between 0.5 and $1.5 \mathrm{mg}$ per kilogramm of body weight per day. If we took over the treatment of children from other doctors and they have already started with other sartan treatment (e.g., losartan, telmisartan) we continued this under the condition of good compatibility. In case of beta blocker treatment indication was mostly insufficient growth inhibition of sinus of valsalvae with valsartan treatment. We extended therapy with metoprolol in young patients and bisoprolol in patients older than 12 years. Atenolol was not given. Carvedilol was the choice in children below 12 years due to the current guidelines. Parents have to give informed consent for medical treatment.

Concerning surgical treatment, criteria for prophylactic 
aortic root replacement in adults are clearly defined but timing in children is more difficult. As soon as aortic dilatation is exceeding $5.5 \mathrm{~cm}$ without risk factors or $5 \mathrm{~cm}$ with risk factors (positive family history for aortic dissection, growth of aortic root greater than $1 \mathrm{~cm} /$ year, dilatation involving ascending aorta, more than mild aortic valve regurgitation, before other than vascular major surgery, women planning pregnancy) a surgical intervention should be commenced in adults (11). Bradley et al. from Canada even recommend aortic root replacement at an aortic root diameter of $5 \mathrm{~cm}$ or a growth of aortic root of $0.5 \mathrm{~cm} /$ year independently from risk factors (12).

In follow-up examinations in childhood the z-score of the aortic root is most important whereas a definite value for an indication for aortic root replacement is missing. A rapid rise of z-score is an indication for surgery. Similar to adults, a prophylactic aortic root replacement in childhood should be commenced with a diameter larger than $5 \mathrm{~cm}$ or growth of the aortic root greater than $0.5 \mathrm{~cm} /$ year (12). Also family history concerning aortic dissection is very important as a prognostic factor (13). Basically aortic root replacement in childhood should be delayed to adolescence whenever possible to avoid valve-patient mismatch later in life. This must of course be balanced to the risk of dissection (14).

Whenever possible, we preferred the valve-sparing aortic root replacement (David procedure).

We also examined transition progress and analyzed problems and barriers to transition. After transition, the specialized adult Marfan Clinic of the cardiology department in University Heart Center Hamburg takes care of the patients.

We used Filemaker software V.10 Pro Advanced to collect data. We performed statistical analysis with Microsoft Excel 2007 and SPSS Statistics 21.0.

Quantitative variables are expressed as means with standard deviation and qualitative data as numbers. We used the unpaired $t$-test to compare quantitative data between groups and considered $\mathrm{P}$ values $<0.05$ as significant.

To assess clinical data, we obtained informed consent from patients or patients' parents.

This study was approved by the Hamburg ethical board (PV 4005).

\section{Results}

Between January 2008 and October 2017, we recruited 424 patients with suspected MFS. The disease was diagnosed according the RGC in 149 patients with a mean age of $9.5 \pm 5.8$ years. Thirty-four patients $(22.8 \%)$ required transition to adult care. We have already transferred 20 patients $(58.9 \%)$ to adult care completely; 12 patients $(35.3 \%)$ are still in the transition process and two patients (5.9\%) have been lost to follow up.

We compared prevalence of dilatation of the sinus of Valsalva, mitral valve prolapse, ectopia lentis, and systemic manifestation in total and in detail in patients younger and older than 18 years (Table 1). Prevalence of skin striae, wrist and thumb sign, and reduced elbow extension was significantly higher in patients older than 18 years and therefore in the transition process. All other clinical features of MFS were more prevalent in older patients, but this difference was not significant. Cardiac pathologies showed an especially high presence in transitioning patients. Dilatation of the sinus of Valsalva occurred especially often $(71 \%)$ in patients in transition process and early with a mean onset of $9.9 \pm 5.8$ years. Systemic pathologies $(11.9 \pm 4.5$ years $)$ and ectopia lentis $(8.3 \pm 5.3$ years $)$ also appeared early and require attention.

In our cohort, in 80 patients medical treatment was started due to cardiovascular abnormalities (dilatation of sinus of Valsalva, severe mitral valve prolapse or regurgitation). Therefore, 61 pediatric patients $(55.7 \%)$ and 21 patients in transition process $(61.8 \%)$ were treated. We initialized pharmacological treatment with a mean age of $10.2 \pm 5.1$ years. In detail, regardless of the status of transition, treatment with sartans was started in 60 patients $(40.5 \%)$ and treatment with beta-blockers in 25 patients $(16.9 \%)$. Fourteen patients $(9.5 \%)$ required a combination of sartans and beta-blockers. One of the sartan-treated patients $(1.7 \%)$ interrupted therapy whereas five patients $(20.0 \%)$ of beta-blocker-treated patients ended therapy because of side effects. The patient who stopped taking sartans showed weakness and lower load capacity in everyday life. Beta blockers were stopped because of headache, weakness with lower load capacity in everyday life and listlessness.

Valve sparing aortic root replacement (David procedure) was necessary in seven pediatric patients with a mean age of $11.5 \pm 5.6$ years. We found no aortic dissection in our patient cohort. In our collective other cardiac surgeries were not necessary.

We saw one boy (16 years) with atrial flutter and need of right atrial ablation. Otherwise we did not see malignant cardiac arrhythmias or severe ventricular dysfunction. 
Table 1 Prevalence of pathologies of MFS in children and young adults

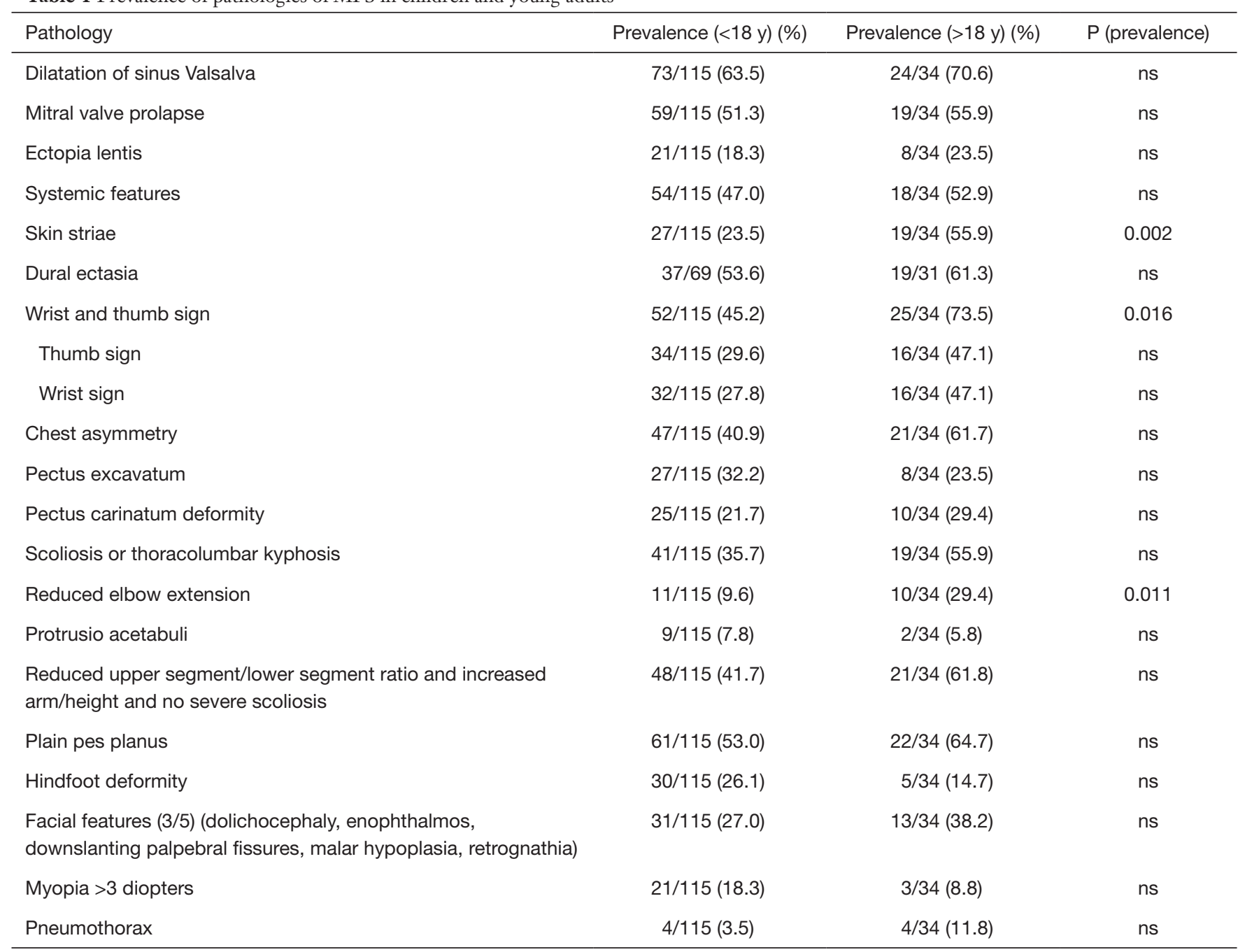

MFS, Marfan syndrome; y, years; ns, not significant.

\section{Discussion}

Because of the possibility of early diagnosis of MFS in childhood, there is a growing cohort of young patients who require a safe and lifelong follow-up regime. In particular, the high prevalence of cardiovascular pathologies in young Marfan patients who are transitioning to adult care shows the relevance of the effectiveness of this transition and the growing need for good transition concepts. Aortic dilatation was already present in $71 \%$ of patients in our patient group in transition process and mean onset was early $(9.9 \pm 5.8$ years). Marfan patients are at a risk of aortic complications and the need for surgical treatment in adulthood (15). The risk of aortic dissection rises with age and causes the death of more than $50 \%$ of undiagnosed and untreated patients before the age of 40 years (16). Being lost to follow-up in the transition process is obviously dangerous. Inadequate follow-up examinations endanger the patient for unrecognized aortic root dilatation and the associated missing timely drug therapy or surgical treatment indication.

Sixty-two percent of transitioning patients were treated with sartans, beta-blockers or both. Equal efficacy of sartans and beta-blockers was shown previously (17-19). However, in our experience, tolerability and compliance concerning taking medication is clearly superior in patients with sartan treatment. Patients who underwent sartan therapy stopped treatment much less frequently $(1.7 \%)$ than those with beta-blocker therapy (20.0\%) due to unwanted side effects. 
The reduced side effects from sartans minimize the daily influence of disease and its necessary medical treatment on patients. Medical treatment at an early age requires the minimum possible side effects for young patients in the interference-prone phase of child growth. Given the results in our patient cohort as well as in current studies it would thus be reasonable to especially treat young Marfan patients with sartans $(17,20)$.

In general the beginning of a medical treatment in childhood is difficult especially concerning convincement of parents. The start of a prophylactic treatment is even harder. As soon as we saw cardiac pathologies, necessary start of treatment was clear for us and the parents. But without presence of cardiac pathologies which is often the case in young children, beginning of a prophylactic treatment was difficult. In case of missing agreement of parents we followed up the patients closely (three to four times a year). We initiated prophylactic medical treatment whenever z-score for rising or z-score was bigger than two.

Prophylactic aortic root repair has the highest impact on surveillance of Marfan patients and shows a much lower morbidity and mortality than emergency aortic root replacement $(9,14)$. Thus it is most important to recognize the right time for aortic root replacement.

Beside the cardiovascular pathologies, we also found a rising prevalence of ectopia lentis and the features of the systemic manifestation of MFS in adolescence. To optimize patient care and guarantee the best outcomes and quality of life for patients, a multidisciplinary team is vital. von Kodolitsch et al. summarized the necessary disciplines to care for patients with MFS: pediatrician/ pediatric cardiologist, cardiologist, human geneticist, heart surgeon, vascular surgeon, orthopedic surgeon, ophthalmologist, nurse, and auxiliary disciplines including forensic pathologist, radiologist, pulmonologist, sleep specialist, rhythmologist, dentist and orthodontist, neurologist, obstetric surgeon, psychologist, and rehabilitation specialist (1). The pediatrician, pediatric cardiologist, pediatric surgeon, pediatric orthopedic surgeon, and pediatric endocrinologist are particularly involved when treating children. Therefore, during the transition process, multiple personnel changes are a risk for lack of compliance and should be monitored by a Marfan specialist. At least the ophthalmologist and dentist do not usually change.

The transition process itself thus comprises a double change of responsibility. On the one side, pediatric cardiologist and other pediatric disciplines hand over patient care to adult specialists. On the other side, the liability for the disease and the involved tasks move from the parents to the children. Parents are no longer responsible for organization and care of disease. Consequently, it is a risky process that is at risk of many problems, especially the danger of being lost to follow-up. In our patient cohort, two patients were lost to follow-up during the transition process. To minimize the hazard of being lost to follow up and to avoid complications, the change of responsibility from pediatric to adult doctors and from parents to the patients should be performed consecutively. Reevaluation of a successful transition process is essential and the final step of pediatric care of chronic diseases like MFS. Even though pediatricians hand over patient care, the patient still has a long way to go and requires lifelong follow-up.

To guarantee the best outcome for patients, medical and personal patient resources are indispensable. Agebased disease education and understanding of disease in the family and of the patient are relevant. Even young children should understand risks of disease to develop. This results in the correct handling and accurate decisions such as choice of sports and later choice of job. The disease then becomes not a prohibition imposed by parents or doctors, but a meaningful restriction. This understanding reduces complications of disease and enables a longer life. Patients must cope with the influence of disease in everyday life. Ocular, skeletal, and cardiovascular features may cause problems and stigmatization of children and later adults (21). Finally, they must cope especially with the potentially lifethreatening consequences of the disease (22). This is one of the most important tasks in the care of pediatric Marfan patients to educate self-responsible adults who no longer need their parents' care.

Consequently, besides the necessary pharmacological and surgical care, psychological care should start in early childhood. Health-related quality of life in adults with MFS is impaired compared with that in children $(23,24)$. Mueller et al. described the lack of awareness of illness, effective social embedding, and mild symptom manifestation during childhood as possible reasons for higher quality of life in pediatric Marfan patients (25). Even though the lack of awareness and milder symptoms present in childhood change as the patient ages, a good quality of life for all Marfan patients should be the highest aim. To preserve valuable quality of life, strengthening of pediatric Marfan patients is necessary to allow good disease coping. After a successful disease education in childhood and acceptance of the chronic illness and need for consistent medical care, 
transition to adult care is easier and safer. Patients should understand the medical care and its multidisciplinary involvement. Therefore, decisions about and consequences for capacity in school, sport, and work life are more natural for patients. Additionally, patients need social support to prevent and reduce behavioral effects such as depression in patients with MFS (26). An effective self-management of patients is indispensable to optimize impact of treatment and outcome (10). Thereby individuals, as well as family, community, and health care systems, influence patients' self-management. Adolescents and young adults are in an especially vulnerable phase of life during which emotional distress is present and lifestyle modifications may influence disease outcomes and adherence to therapy. To develop self-consciousness and coping skills family, community, and particularly peer support are essential and should be respected and addressed in the care of Marfan patients.

\section{Conclusions}

We conclude: first, the prevalence of clinical signs increases during childhood and reaches a climax at the time of transition to adult care. Dilatation of the aortic root in particular requires pharmacological and sometimes also surgical attention. Sartans and beta-blockers are effective; compliance was better with sartan treatment in our patient cohort because there were fewer side effects. Second, the whole variety of clinical Marfan features is already present in this patient group of young adults. Consequently, close pediatric surveillance is indispensable in childhood and, in addition to child-centered care, specialized treatment and multidisciplinary follow-up examinations in different pediatric medical disciplines are essential. Third, the transition process itself is risky for patients, their family, and accompanying doctors. Consequently, patients need early disease education and psychological support to help patients to cope with their disease and adapt their lives accordingly. A good quality of life and long-term outcomes are most important for the care for Marfan patients. Early diagnosis is thus a challenge and a chance at the same time. Ongoing development of preventive strategies for Marfan patients, pharmacologically and psychologically, should therefore remain the highest aim.

\section{Acknowledgements}

We thank the Edanz group (http://www.edanz editing.com/ac) for editing a draft of this manuscript.

\section{Footnote}

Conflicts of Interest: The authors have no conflicts of interest to declare.

Etbical Statement: This study was approved by the Hamburg ethical board (PV 4005). To use patient data, we obtained informed consent of patient, parent or legal guardian. Additional informed consent was obtained from all individual participants for whom identifying information is included in this article. All procedures performed in studies involving human participants were in accordance with the ethical standards of the institutional and/or national research committee and with the 1964 Helsinki Declaration and its later amendments or comparable ethical standards.

\section{References}

1. von Kodolitsch Y, Rybczynski M, Vogler M, et al. The role of the multidisciplinary health care team in the management of patients with Marfan syndrome. J Multidiscip Healthc 2016;9:587-614.

2. von Kodolitsch Y, De Backer J, Schüler H, et al. Perspectives on the revised Ghent criteria for the diagnosis of Marfan syndrome. Appl Clin Genet 2015;8:137-55.

3. Judge DP, Dietz HC. Marfan's syndrome. Lancet 2005;366:1965-76.

4. Eidt D, Frank M, Reimann A, et al. Maßnahmen zur Verbesserung der gesundheitlichen Situation von Menschen mit Seltenen Erkrankungen in Deutschland. Forschungsbericht [Actions to improve the health situation of people with rare diseases in Germany. A research report. Study on behalf of the federal ministry of health]. Studie im Auftrag des Bundesministeriumsfür Gesundheit, 2009. Available online: http://www.bmg.bund.de/themen/ praevention/gesundheitsgefahren/seltene-erkrankungen. html, accessed February 23, 2010. German.

5. Loeys BL, Dietz HC, Bravermann AC, et al. The revised Ghent nosology for the Marfan syndrome. J Med Genet 2010;47:476-85.

6. Mueller GC, Stark V, Steiner K, et al. The Kid-Short Marfan Score (Kid-SMS) - an easy executable risk score for suspected paediatric patients with Marfan syndrome. Acta Paediatr 2013;102:e84-9.

7. Dietz HC. Marfan Syndrome. 2001. In: Pagon RA, Adam MP, Ardinger HH, et al. editors. GeneReviews. Seattle 
(WA): University of Washington, Seattle, 1993-2015.

8. Murdoch JL, Walker BA, Halpern BL, et al. Life expectancy and causes of death in the Marfan syndrome. N Engl J Med 1972;286:804-8.

9. Pyeritz RE. Marfan syndrome: 30 years of research equals 30 years of additional life expectancy. Heart 2009;95:173-5.

10. Modi AC, Pai AL, Hommel KA, et al. Pediatric selfmanagement: a framework for research, practice, and policy. Pediatrics 2012;129:e473-85.

11. von Kodolitsch Y, Robinson PN. Marfan syndrome: An update of genetics, medical and surgical management. Heart 2007;93:755-60.

12. Bradley TJ, Alvarez NAM, Horne SG. A Practical Guide to Clinical Management of Thoracic Aortic Disease. Can J Cardiol 2016;32:124-30.

13. Roman MJ, Rosen SE, Kramer-Fox R, et al. Prognostic significance of the pattern of aortic root dilatation in the Marfan syndrome. J Am Coll Cardiol 1993;22:1470-6.

14. Keane MG, Pyeritz RE. Medical management of Marfan syndrome. Circulation. 2008;117:2802-13.

15. Milewicz DM, Dietz HC, Miller DC. Treatment of aortic disease in patients with Marfan syndrome. Circulation 2005;111:e150-7.

16. Ammash NM, Sundt TM, Connolly HM. Marfan syndrome-diagnosis and management. Curr Probl Cardiol 2008;33:7-39.

17. Milleron O, Arnoult F, Ropers J, et al. Marfan Sartan: a randomized, double-blind, placebo-controlled trial. Eur Heart J 2015;36:2160-6.

18. Lacro RV, Dietz HC, Sleeper LA, et al. Atenolol versus

Cite this article as: Stark VC, Doering K, von Kodolitsch Y, Kozlik-Feldmann R, Mueller GC, Olfe J, Rybczynski M, Schueler H, Mir TS. The transition of pediatric Marfan patients to adult care: a challenge and its risks. Cardiovasc Diagn Ther 2018;8(6):698-704. doi: 10.21037/cdt.2018.09.13 losartan in children and young adults with Marfan's syndrome, N Engl J Med 2014;371:2061-71.

19. Mueller GC, Stierle L, Stark V, et al. Retrospective analysis of the effect of angiotensin II receptor blocker versus $\beta$-blocker on aortic root growth in paediatric patients with MFS. Heart 2014;100:214-8.

20. Gao L, Chen L, Fan L, et al. The effect of losartan on progressive aortic dilatation in patients with Marfan's syndrome: a meta-analysis of prospective randomized clinical trials. Int J Cardiol 2016;217:190-4.

21. Peters K, Apse K, Blackford A, et al. Living with Marfan syndrome: coping with Stigma. Clin Genet 2005;68:6-14.

22. Speed TJ, Mathur VA, Hand M, et al. Characterization of pain, disability, and psychological burden in Marfan syndrome. Am J Med Genet A 2017;173:315-23.

23. Rand-Hendriksen S, Johansen H, Semb SO, et al. Healthrelated quality of life in Marfan syndrome: a crosssectional study of Short Form 36 in 84 adults with a verified diagnosis. Genet Med 2010;12:517-24.

24. Velvin G, Bathen T, Rand-Hendriksen S, et al. Satisfaction with life in adults with Marfan syndrome (MFS): associations with health-related consequences of MFS, pain, fatigue, and demographic factors. Qual Life Res 2016;25:1779-90.

25. Mueller GC, Steiner K, Wild JM, et al. Health-related quality of life is unimpaired in children and adolescents with Marfan syndrome despite its distinctive phenotype. Acta Paediatr 2016;105:311-6.

26. Moon JR, Cho YA, Huh J, et al. Structural equation modeling of the quality of life for patients with marfan syndrome. Health Qual Life Outcomes 2016;14:83. 